\title{
Selenium Intakes in Irish Adults
}

\author{
M. Buffini ${ }^{1}$, J. Ryan ${ }^{1}$, A.P. Nugent ${ }^{1,2}$, J. Walton ${ }^{3}$, A. Flynn ${ }^{3}$ and B.A. McNulty ${ }^{1}$ \\ ${ }^{1}$ UCD Institute of Food and Health, School of Agriculture and Food Science, University College Dublin, Belfield, \\ Dublin, Ireland, ${ }^{2}$ Institute for Global Food Security, Queens University Belfast, N. Ireland and ${ }^{3}$ School of Food and \\ Nutritional Sciences, University College Cork, Cork, Ireland.
}

Selenium (Se) is an essential trace element which has an important role as a constituent of seleno-proteins. These proteins are involved in various physiological processes including thyroid hormone metabolism, DNA synthesis, protection from oxidative damage, fertility and reproduction ${ }^{(1)}$. The aim of this study was to estimate current intakes and food sources of Se by Irish adults and to assess the prevalence of inadequate intakes. Analyses were based on data from the National Adult Nutrition Survey (2008-2010) (www.iuna. net). A 4-day semi-weighed food diary was used to collect food and beverage intake data from a nationally representative sample of Irish adults aged 18-90 years (n 1500). The selenium content of foods and drinks was predominantly determined using Se data from the Total Diet Study (TDS) ${ }^{(2)}$ For certain foods where Se values were not available, the UK food composition tables ${ }^{(3)}$ and the US food composition tables ${ }^{(4)}$ were used to assign suitable values. SPSS C V22 was used to calculate mean daily intake (MDI) and the percentage of the population not meeting Se recommendations.

\begin{tabular}{|c|c|c|c|c|c|c|c|c|c|}
\hline & \multirow{2}{*}{$\begin{array}{c}\text { Total Population } \\
18-90 \mathrm{y} \\
(\mathrm{n}=1500)\end{array}$} & \multicolumn{4}{|c|}{ Males } & \multicolumn{4}{|c|}{ Females } \\
\hline & & $\begin{array}{c}18-35 y \\
(n=276)\end{array}$ & $\begin{array}{c}36-50 y \\
(n=205)\end{array}$ & $\begin{array}{c}50-64 \mathrm{y} \\
(\mathrm{n}=153)\end{array}$ & $\begin{array}{c}\geq 65 y \\
(n=106)\end{array}$ & $\begin{array}{c}18-35 y \\
(n=255)\end{array}$ & $\begin{array}{c}36-50 y \\
(n=232)\end{array}$ & $\begin{array}{c}50-64 y \\
(n=153)\end{array}$ & $\begin{array}{c}\geq 65 y \\
(n=120)\end{array}$ \\
\hline$\overline{\text { Mean }(\mu \mathrm{g} / \text { day })}$ & 71.7 & $86 \cdot 3^{\mathrm{a}}$ & $76 \cdot 8^{\mathrm{b}}$ & $77 \cdot 4^{\mathrm{b}}$ & $74.9^{\mathrm{b}}$ & 63.8 & $62 \cdot 3$ & $65 \cdot 2$ & $62 \cdot 3$ \\
\hline $\mathrm{SD}$ ( $\mu \mathrm{g} /$ day) & $30 \cdot 2$ & $34 \cdot 1$ & $23 \cdot 8$ & $24 \cdot 3$ & $28 \cdot 5$ & $26 \cdot 1$ & $24 \cdot 4$ & $33 \cdot 1$ & $36 \cdot 1$ \\
\hline P5 ( $\mu \mathrm{g} /$ day) & $35 \cdot 5$ & $44 \cdot 2$ & $47 \cdot 5$ & $43 \cdot 4$ & $40 \cdot 3$ & $29 \cdot 5$ & $34 \cdot 1$ & $33 \cdot 3$ & $31 \cdot 1$ \\
\hline P50 ( $\mu \mathrm{g} /$ day) & $66 \cdot 9$ & $81 \cdot 1$ & $74 \cdot 4$ & $43 \cdot 4$ & $71 \cdot 1$ & $59 \cdot 3$ & $56 \cdot 9$ & $59 \cdot 1$ & $54 \cdot 3$ \\
\hline P97.5 ( $\mu \mathrm{g} /$ day) & $148 \cdot 2$ & $167 \cdot 9$ & $138 \cdot 8$ & $43 \cdot 4$ & $156 \cdot 9$ & $126 \cdot 0$ & $119 \cdot 5$ & $139 \cdot 0$ & $54 \cdot 3$ \\
\hline Percentage below AI & $55 \cdot 0$ & $31 \cdot 2$ & $42 \cdot 9$ & $42 \cdot 5$ & $47 \cdot 2$ & $64 \cdot 7$ & $72 \cdot 4$ & $71 \cdot 2$ & $78 \cdot 3$ \\
\hline
\end{tabular}

Adequate intake (AI) for Se $70 \mu \mathrm{g} / \mathrm{d}^{(5)}$. Differences between age groups were analysed with a one-way ANOVA. Superscript letters denote statistical significance $(\mathrm{p}<0 \cdot 01)$.

MDI of Se in the total population was $71.7 \mu \mathrm{g} / \mathrm{d}$, with significantly higher intakes reported in men $(80 \cdot 2 \mu \mathrm{g} / \mathrm{d})$ compared to women $(63.4 \mu \mathrm{g} / \mathrm{d}, \mathrm{p}<0.01)$ however, when adjusted for energy, results showed that Se intakes were higher in women ( $<<0.01)$. Meat $\&$ meat products were the main contributor to Se intakes for both males and females at (37\%) and (31\%) respectively, this was followed by fish \& fish dishes at (11\%) and (13\%) respectively. Overall, 55\% of Irish adults aged 18-90 years were not meeting the AI for Se, and when spilt by gender a higher proportion of women $(76 \%)$ compared to men $(39 \%)$ did not meet this recommendation. Further investigation is required to invest Se status and its impact on overall health.

This study was funded by the Irish Department of Agriculture, Fisheries and Food under the Food for Health Research Initiative (2007-2012).

1. Rayman M (2002) Lancet 356 pp. 233-241.

2. Food Safety Authority of Ireland (2016) Available at: https://www.fsai.ie/publications_TDS_2012-2014/

3. Public Health England (2015) PHE. London.

4. USDA (2015) National Nutrient Database for Standard References 28 https://ndb.nal.usda.gov/ndb/

5. EFSA (2014) NDA Panel Available at: http://onlinelibrary.wiley.com/doi/10.2903/j.efsa.2014.3846/epdf 